VOLUMEN 42

Fasc. 1 Pag. 1-160, edit.: 6-7-1973

Fasc. 2-3 Pag. 161-368, edit.: 15-8-1973

Fasc. 4 Pag. 369-540, edit. : 28-9-1973

\title{
INDEX AUTORUM
}

Abdelmalik, W. E. Y., 3

Alvis, C., 285

Armitage, K. B., 295, 491

Aston, R. J., 225

BAKKER, C., 157

Balakrisnan NaIr, N., 413

BICK, H., 393

Borodich, N. D., 527

Ciereszko, L. S., 77

Czeczuga, B., 85, 355

Deelder, C. L., 364

Drews, E. F., 393

Duthie, H. C., 161

El-Shinawy, R. M. K., 3

ERTL, M., 429

FALCONER, A. C., 45

George, P. V., 31

Glooschenko, W. A., 285

GradzKI, F., 85

HANGOGK, F. D., 243

Havlena, F. K., 527

IsHAK, M. M., 3

KALBE, L., 21

KECK, R., 369

Keeney, D. R., 509

Krisna Pillai, N., 143, 403

LAMINGER, H., 153

LANDE, A., 335
Mahmoud, K. A., 3

MARshall, B. E., 45

Maurer, D., 369

Metwalli, A. M., 451

Miron, I., 345

Mohsen, A. F., 1, 451

Nadakal, A. M., 21

NAGELL, B., 461

NASR, A. H., 451

Nichols, D. S., 509

Peelen, R., 540

Perkins, D. L-. 77

Pravda, O., 97

SAlomé, B. Z., 363

Saxena, B., 295, 491

SchönboRn, W., 63

Sebastian, M. J., 143

Shahul Hameed, M., 403

Singh, U. P., 445

SkAlski, B., 355

Sreenivasa, M. R., 161

Tomajka, J., 429

UTRECHT, W. L. van, 365

VAAS, K. F., 158, 159, 366, 368

VENKATAKRISNAN, R., 413

WATLing, L., 369

WOLFF, W. J., 381

Zelinka, M., 13 